\title{
Project SUE as a learning experience
}

\author{
by K. C. SEVCIK, J. W. ATWOOD, M. S. GRUSHCOW, \\ R. C. HOLT, J. J. HORNING and D. TSICHRITZIS \\ University of Toronto \\ Toronto, Ontario, Canada
}

\section{INTRODUCTION}

"It is absurd to separate the study of designing from the practice of design." (Christopher Alexander)

Project SUE at the University of Toronto is developing an operating system for the IBM System/360 family of computers. We are basing our work as much as possible on previous research in operating systems, including that of Dijkstra, ${ }^{1,2}$ Lampson, ${ }^{3}$ Brinch Hansen, ${ }^{4}$ and the MULTICS group. ${ }^{5-7}$ Their ideas have been tested in actual systems, but separately, and on uncommon machines. We wish to combine their ideas and the ideas of others in a system for a widely available machine.

In Project SUE, we have attempted not only to build an operating system, but also to learn how to organize a large software project. To this end, we have attempted to structure and document the project itself as well as the system.

We have set high standards for the system. We want it to be efficient. We want it to be extensible in the sense that it is possible to append various protected subsystems which each serve a community of users. Above all, we want the system to be reliable and understandable. We make no attempt to compete with generality of existing operating systems. Rather, we are creating an operating system nucleus (in the sense of Brinch Hansen ${ }^{4}$ ) which can be extended to support particular applications. The system nucleus is designed to support simultaneously, for example, an interactive system and an independent batch monitor.

This paper presents our objectives, and how they have influenced our selection among reasonable alternatives in some design decisions. This material has not arisen from abstract discussions of the theory of operating systems. It comes from the specification, detailed design and partial implementation of a system. A description of the system design is available elsewhere. ${ }^{8-9}$
Here, we will only discuss some aspects of the design process.

\section{SYSTEM STRUCTURE}

The concept of processes has been useful in understanding and designing operating systems.2,4,7,10 Each process proceeds asynchronously as if executing on its own (virtual) machine, except when mechanisms for the explicit interaction of processes are invoked. These interactions may depend on relationships among the processes. For example, processes may be

(1) regarded as equals, or

(2) related in a tree-structured hierarchy.

The latter situation arises if each process (after the first one) is created by another process. In what we call the creation tree, a process is a son of the process which created it, and the father of any process which it creates. While equality among processes is a simple relationship, the weak system structure it imposes makes other goals (understanding the system, assuring its reliability, and establishing its correctness) difficult to attain. A hierarchy among the processes specifies a logical order in which to understand them and demonstrate their correctness.

The virtual machine upon which a process executes is defined by the set of operations supported for the process by other processes, lower level software, and hardware. Each process should be sufficiently simple that its correct operation can be demonstrated (on the assumption that its virtual machine operates correctly). If the correct operation of a virtual machine does not depend on the correctness of any process using it, then a logical order for understanding and demonstrating the correctness of the system is apparent. 
It is possible to distinguish between types of process hierarchies in which virtual machines are

(1) completely ordered,

(2) partially ordered.

In the former case, the system is viewed as a sequence of increasingly sophisticated virtual machines ("onionlike layers"). The lowest level virtual machine corresponds to the hardware, and each higher level is created by adding a layer of software (possibly composed of processes) to the previous virtual machine. Dijkstra has described the T.H.E. system in terms of six levels of virtual machines. ${ }^{2}$ Every process in such a system is associated with the level of virtual machine upon which it executes. When virtual machines are completely ordered, any two processes are related in one of two ways; either they have the same virtual machine, or one of them helps provide the virtual machine used by the other. A more general process hierarchy results when virtual machines are only partially ordered. Independent processes then need not have the same virtual machine.

The SUE system structure permits hierarchies of the latter type although several onion-type layers are distinguishable and worthy of mention. The Kernel is a layer of software which uses the hardware to implement processes (their creation, destruction and communication), protection, simple management of memory and channels, and timing facilities. The innermost group of processes uses the Kernel to create the Nucleus, a more sophisticated virtual machine which provides disk files, peripheral input and output facilities, and mechanisms for measurement and accounting. The virtual machines used by the processes which form the Nucleus can be partially ordered with respect to sophistication. It is possible to create a set of virtual machines which are completely ordered by adding to some of the virtual machines facilities which will not be used by the corresponding processes. This corresponds to imposing an onion-type hierarchy on the processes in the Nucleus.

There are many ways of grouping operating system activities to form processes. At one extreme each conceptually asynchronous activity might be carried out by a separate process. However, asynchronism among activities does not necessarily justify the existence of several processes with frequent interactions among them. For example, at one time we planned to have a process to manage each disk spindle and each disk control unit. However, each disk spindle manager would interact with a control unit manager so frequently that little asynchronous activity would occur. We have concluded that the extra processes are not justified and that all disk spindles and all disk control units should be managed by a single process. Other aggregations of activities have been adopted, and the SUE Nucleus now consists of only seven processes.

\section{COMMUNICATION AND COOPERATION AMONG PROCESSES}

Because our operating system is based on the cooperation of processes, the communication mechanisms must be efficient, easy to understand, and easy to use, but secure from unauthorized use and the danger of deadlock. ${ }^{12,13,14}$ Many schemes for process interaction have been developed. Each is based on either

(1) shared data, or

(2) message passing.

Process interactions in Dijkstra's T.H.E. system use shared data called semaphores and special indivisible operations for manipulating them. ${ }^{1}$ In a message passing scheme suggested by Wulf, each process possesses a number of ports, and each port is the interface to a communication link with another process..$^{15}$ Establishing the communication link may be an expensive operation, but, with the link established, less checking is necessary as each message is passed. A mailbox with several message slots may be inserted in a communication link to provide automatic buffering of messages. ${ }^{16}$ Because we desire autonomy among processes in Project SUE, a message passing form of communication is more appropriate than a scheme based on shared data.

Message passing through ports and mailboxes was initially accepted as the mechanism for interprocess communication in Project SUE. Much work was done examining protocols for establishing and using communication links, and making sure that deadlock would not occur. ${ }^{16}$ The scheme seemed to be compatible with our goals of making the system efficient and understandable.

Only after much more time and thought did we identify some problems with communication through ports and mailboxes alone. One problem was establishing the communication links. A process must name the process, or class of processes, with which it wishes to communicate. Unless system-wide standard names are established, another significant communication mechanism is required to coordinate the naming of processes. 
Processes which provide a service, such as device allocation or file system management have special communication requirements. A large number of processes wish to use each such service. With extensibility as a design goal, the number of processes that can simultaneously have a communication link to a particular service process should not be limited (although such limits exist in many systems). Providing each service process with enough ports to guarantee that competition could not lead to deadlock would require the commitment of an excessive amount of memory. We considered adding a second form of mailbox which could attach a single port of a service process to an unbounded number of other ports. However, the complexity of such objects and new problems in their design compelled us to seek a better solution.

Mailboxes are not appropriate for passing large messages (such as block transfers on input and output). Not only is memory space committed unnecessarily to mailbox buffers, but each message must be moved twice (source to mailbox buffer, then mailbox buffer to destination) when one move could suffice. Finally, more careful analysis indicated that passing even small messages through mailboxes would not be as efficient as we had hoped.

Another mechanism was suggested to supplement communication through mailboxes. We call service processes facilities and they are used as are the monitors described by Hoare. ${ }^{17} \mathrm{~A}$ requesting process contacts a facility directly by issuing a facility call. The call is unbuffered; and the requesting process cannot proceed until the facility completes the requested service.

For the sake of system structure, we restrict which processes are allowed to call on any particular facility. A straightforward mechanism for representing this information is an access matrix, whose $(i, j)$ th element indicates whether process $i$ may call upon facility $j$. However, such a matrix is not easily kept current in an environment where processes are dynamically created and destroyed. Also the access matrix does not contribute to system structure.

By relating permissibility of facility calls to the creation tree, a hierarchical system structure may be enforced implicitly. Three alternatives we considered are that a facility may be called upon

(1) only by its descendants,

(2) only by its descendants, and by its younger brothers and their descendants, or

(3) only by processes farther from the root of the creation tree.

The second of these represents a compromise between the other two. The first is the simplest and most understandable. The advantage of the second alternative over the first is that processes which provide a facility are not compelled to also create and monitor sons. The third alternative was rejected because the second represents a more structured solution which does not greatly impair flexibility. Choosing between the first and second alternatives was difficult. After several weeks of debate, expediency of implementation caused us to permit facilities to be called upon only by their descendants (first alternative).

The facility call mechanism solves the problems of naming the process to be contacted and of allowing service processes to respond to arbitrarily many customers. We soon realized that facility calls were also sufficient for all other communication needs in the SUE system. Conversations between any two processes are accomplished by facility calls from each to one of their common ancestors. Service requests can be buffered by creating a son to provide the buffering. Thus, in order to reduce the number of different system objects and mechanisms, mailboxes and ports have been eliminated from the SUE System. All interprocess communication is done with the facility call mechanism.

Because all facility calls are directed toward the root of the creation tree, deadlock can be prevented by assuring that each facility completes each user request within finite time. Certain situations require that facility calls be used in an unusual manner. The innermost Nucleus processes are situated in the system structure where disk files and typewriter communication are not available. Yet they need to report error conditions to the operator, and record accounting and measurement information in disk files. Thus the innermost Nucleus processes occasionally require the assistance of a descendant which is at a level of the process hierarchy where disk files and operator communication are available. This descendant, known as the Special Condition Manager, effectively provides service to its ancestors in the creation tree. So that no process waits for a descendant, the facility call mechanism is employed as follows: The Special Condition Manager creates a son for each ancestor which may require its services. Each son issues a facility call on the corresponding ancestor, requesting the next "special condition." When a process wishes service from the Special Condition Manager, it simply completes the service call (which should be outstanding) indicating what "special condition" exists. If the son of the Special Condition Manager has not issued the facility call, the process must not wait for the call to occur, but must take some alternative action. Thus, information may be lost if the Special Condition Manager does not react 
with sufficient speed, but special conditions cannot deadlock the system.

\section{AUTHORIZATION, ACCOUNTING AND MEASUREMENT}

An operating system should provide mechanisms to prevent unauthorized and excessive use of system resources. It should also be able to measure resource usage and attribute it to processes or groups of related processes.

The central mechanism in the authorization and accounting functions of the SUE system is the concept of capabilities. ${ }^{5,18,19}$ A capability is a control block associated with a process which indicates that the process is authorized to use a particular resource in a particular manner. Processes are not allowed to tamper with the information held in capabilities (especially their own!). The ability to create and modify capabilities is restricted to a carefully protected routine deep in the Kernel.

Capabilities are but one possible way of representing protection information. Lampson has described a theory of protection based on objects (resources and processes) and domains. ${ }^{3}$ A process executes in a particular domain. A domain is defined by the manner in which processes executing in that domain are authorized to use the objects of the system. When processes are units of protection as well as units of asynchronous activity, the domain of a process may be represented as a list of capabilities, one for each resource accessible by the process.

An alternative manner of representing protection information is to associate with each resource a list of processes authorized to use it. This method is less desirable in the SUE system because facilities do not discriminate among processes in providing service. Using the capability representation allows each process to allocate the right to use a facility among itself and its sons.

In the SUE system, we distinguish three varieties of capabilities. One governs resource usage qualitatively (permission to use a disk drive or to access a particular file), while another governs quantitatively (the number of files which may be created or the number of file read operations which may be done). A qualitative capability is known by the Kernel to contain a word of Boolean information representing access rights, while a quantitative capability is known to contain a number. The number may be decremented by the facility whenever the capability is used to request use of resource. This is similar to punching a "meal ticket" each time a meal is consumed. When the count reaches zero, the capability no longer has value. The third variety of capability contains information which is interpreted not by the Kernel, but only by the process which created the capability.

We have chosen not to use two features of general capability schemes. First, we do not allow rights to a resource to be passed between arbitrary processes by transferring a capability. In order to maintain system structure and to avoid difficult questions about how to dispose of leftover capabilities when a process is destroyed, we have restricted all capability transactions to occur between either father and son or facility and user. Second, we do not use capabilities to represent authorization information about some resources needed by every process (such as processor time, and memory space). For resources common to all processes, we use an efficient encoding of authorization information. The routines for manipulating capabilities would be made too complex if they had to deal with each special encoding, so the capability concept is not used for these resources.

During much of the design of the SUE system, we believed that capabilities would have to be associated with longer-lived entities than processes. Consider a permanent disk file which is created by one process, then used from time to time by other processes. The succession of processes each must have a capability for accessing the disk file, yet their life spans need not overlap. We faced the problems of how to keep the capabilities in the system and recognize which capabilities should be given to a particular new process. We planned to have permanent entities, called sponsors, whose capabilities would be kept in disk files. Sponsors correspond more or less to people who pay for use of the computer system. Each process which deals with a permanent file will have been initiated on behalf of some person (sponsor). Thus, each disk file capability could be associated with a sponsor, and transferred, upon request, to processes created later on behalf of that sponsor.

Further investigation revealed difficulties with implementing sponsors within the Nucleus. First, it seemed necessary to give the power of transforming data into capabilities to a process whose virtual machine provided disk files, yet we wished to use capabilities to protect disk files. Second, the scheme would increase the complexity of the flow of capabilities in the system. At process creation, capabilities would come not only from the father, but also from the sponsor. Worse yet, at process destruction, a decision mechanism would be needed to determine which of the remaining capabilities were to be returned to the sponsor and which to the father. 
We have since found an alternative solution to the problem of capabilities for permanent disk files. Since disk space is to be allocated among the independent sub-systems being supported by the Nucleus, and then subdivided by each among its sons, the responsibility for associating subdivisions of file space with people (or sponsors) can be left to each process which divides its space among its sons. Further, by requiring that each suballocation consist of a subset of the father's file space, the father is able to retain, in a single capability, the authorization to the entire file space which it controls. Only when a subsystem process is destroyed is there still a problem of where to keep the file capability. By requiring the number of independent sub-systems to be bounded and small, we can store the file space capability for each sub-system within the Nucleus. This approach eliminates the problem of transferring capabilities to and from peripheral. storage and moves the sponsor structure completely outside the Nucleus. The complexity of a disk resident sponsor structure makes such a move desirable.

Mechanisms for accounting and measurement of resource usage are implemented using capabilities. Essentially, every process is held accountable for the resource usage represented by any capability it receives from its father or a facility. If it does not wish to be financially responsible for the resource usage of its sons, it must record the value of the capabilities passed to each son and the value of the capabilities returned when the son is destroyed. In this manner, resource usage can be attributed to individual processes at as many levels of the system as is desired.

Perhaps the strongest motivation for using capabilities as the mechanism for authorization and resource allocation is that we wish the system to be conveniently extensible. Since we cannot define the universal resource set, we have provided mechanisms so that processes can define arbitrary resources and can authorize and account for their usage.

\section{RELIABILITY AND EXTENSIBILITY}

Our original proposal contains the sentence, "A design criterion is that neither the erroneous nor the malicious program shall be able to 'crash' any other user, or the system, under any combination of circumstances." We initially interpreted this as, "No process should ever have to put itself at the mercy of another process." By our selection of system structure and mechanisms for resource allocation and authorization, we have designed a system in which no process can cause incorrect operation of any process which contrib- utes to the support of its virtual machine (that is, any process closer to the Kernel in the creation tree). Further, two processes on different branches of the creation tree cannot interfere with each other. Thus protection in the SUE system provides two-way insulation ("firewalls") between independent, non-interacting processes, and one-way insulation of processes from their descendants in the creation tree. Every process can be mistreated by any facility from which it requests service, and it has no protection against the whims of its father. However, we do not feel that it is a compromise to our original goal to require a process to trust the virtual machine upon which it runs. Rather, we have learned more precisely what our original goal was and how inadequately defined the terms "user" and "system" are.

Because extensibility is among our goals, we are unwilling to establish a clear distinction between "system" processes and "user" processes. Definition of "user" is always relative to a particular process. The descendants of any process form the set of potential users of that process. All mechanisms within the Nucleus are intended to be understandable and flexible. They may be helpful to subsystems which are appended to the Nucleus. We hope subsystems will exploit the mechanisms provided within the Nucleus. However, subsystems may choose to conceal the Nucleus mechanisms from their descendants. For example, the distinction among the varieties of capabilities, or, in fact, even the existence of capabilities could be concealed by a subsystem from its users. Similarly, the communication mechanism used within the Nucleus can be replaced. A subsystem might, for example, choose to implement mailboxes as the mechanism for communication among its users.

\section{IMPLEMENTATION LANGUAGE AND PROGRAMMING}

Our desire to make the SUE system understandable, modifiable, and extensible along with our desires to facilitate coding and demonstrate correctness made the use of assembly language for implementation unacceptable. Although several systems programming languages have been developed, ${ }^{20,21,22,23}$ we chose to use an available compiler generator ${ }^{24}$ to design and implement a system language specifically for SUE. This language is documented elsewhere. $8,9,25,26$ It features convenient definition of new data types and control structures which facilitate writing understandable programs.

Hoare's first thesis on the use of high level languages in constructing large programs states: ${ }^{27}$ 
"Programming languages are little help in the construction of large programs.

1. To design a 'language' as part of design and implementation of a big system is essential.

2. To 'implement' this language is disastrous.

3. To use a language designed and implemented for any other special purpose is of doubtful benefit."

Hoare's thesis is a valuable warning of potential danger, but our experience indicates that disaster is not inevitable. Language design and implementation indeed absorbed more project resources than was anticipated. However, the benefits of a well-designed, high-level language are being felt in both coding and validation.

The technique of structured programming has been used successfully in the implementation of several systems. ${ }^{2,28,29}$ We have found that the use of structured programming eases the transition from design to coding, and facilitates attempts to demonstrate the correctness of the system.

\section{PROJECT MANAGEMENT}

Our goal in Project SUE has been not only to build an operating system, but also to learn about the process of building operating systems. For this reason, we have generated extensive documentation of what the system design is and how it came to be that way. A large (and growing) workbook incorporates project history, project status, problems as they are discovered, solutions as they are proposed, and decisions as they are made. The development of this paper has been based on material contained in the project workbook.

At intervals of about four months, we have written project evaluation reports (Checkpoint Reports). At each checkpoint, we have thought about how well we are progressing, how well we are fulfilling our goals, and whether some redirection of the project is needed. In the narrow view, Checkpoint Reports interrupt our technical progress for periods from three days to three weeks. In broader perspective, Checkpoint Reports have forced us to periodically reevaluate our goals and priorities. Without scheduled Checkpoints, it is unlikely that we would devote enough attention to these topics.

Technical decisions have been made in a democratic way among as many as six people. Democracies tend to progress slowly, but once all parties are convinced of a decision, confidence in the decision is often greater than if the decision had been made by an individual.

Some particularly difficult decisions have been resolved by selecting a reversible decision. The questions could not be completely investigated in the time we were willing to keep a decision pending, so we assured ourselves that the decision taken would not have such broad impact that the decision could not be reversed with reasonable effort.

Although we intended to use "existing technologies", design time, not programming time, has been our scarce resource. Two reasons for this have been that most of the designers could not devote full time to the project; and that the system language speeds programming.

\section{CURRENT STATUS}

This paper is based on our experience during the first fifteen months of Project SUE. As of July, 1972, we have designed a systems implementation language and implemented a subset sufficient for developing the SUE Kernel and Nucleus. The system structure and all mechanisms for process interaction have been designed and their manner of use documented. The primitive operations provided by the Kernel are designed and are being implemented. Some Kernel modules have been demonstrated correct. Most Nucleus processes are designed and are being implemented. We are encouraging students to create diverse subsystems to be run under the SUE Nucleus.

\section{CONCLUSION}

We started Project SUE with the intention of building a reliable, hierarchical, extensible system in which no distinction is made between "user" process and "system" processes. We needed a compatible set of convenient, structured mechanisms for control, communication, authorization, and accounting. We knew of "existing technologies" for handling each of these problems individually, but not of a unified set of mechanisms which treated all the problems. We underestimated the conceptual design effort involved in modifying the existing technologies to make them mutually compatible and appropriate for an extensible system. Most of the systems from which we have drawn ideas were successful at least in part due to their limited goals. We have slowly become aware that our original goals were very ambitious.

A notable change in our approach has occurred since the start of the project. Initially, we designed the most general mechanisms which were implementable. Recently, we have designed the most restricted mechanisms which would satisfy our needs. Partly this is because we now have a much sharper picture of what 
our needs are. But, also, it reflects a trend toward practicality.

The change in approach can be observed in several areas. The System Language was fully designed early in the project. It has become apparent that much of the generality of the language was costly to implement without contributing greatly to the goals of the project. Early in the project, mailboxes and capabilities in their general sense appealed to us. Both are flexible, powerful, and expensive mechanisms. Recently, we have realized that we can make the system structured and understandable, by using capabilities in a more constrained manner, and using a more restrictive communication mechanism.

It is important to the future of the "Theory of Operating Systems" that new work make use of the knowledge of previous successes and failures in the area. It is also important to test in full-scale systems the adequacy of ideas presented initially at conceptual or philosophical levels. Project SUE has attempted to do both. Frequent introspection has also allowed us to extract some knowledge of designing from our process of design.

\section{ACKNOWLEDGMENTS}

Project SUE is supported by the National Research Council of Canada through the Computer Systems Research Group at the University of Toronto.

\section{REFERENCES}

\section{E W DIJKSTRA}

Cooperating sequential processes

in programming languages (ed. F GENUYS) Academic Press 1968 pp 43-112

2 E W DIJKSTRA

The structure of the T.H.E. multiprogramming system Communication of the ACM Vol 11 No 51968 pp 341-346

3 B W LAMPSON

Dynamic protection structures

Proceedings of AFIPS FJCC Vol 351969 pp 27-38

4 P BRINCH HANSEN

The Nucleus of a multiprogramming system

Communications of the ACM Vol 13 No 41970 pp 238-241

$5 \mathrm{~J}$ B DENNIS E C VANHORN

Programming semantics for multiprogrammed computation

Communications of the ACM Vol 9 No 31966 pp 143-155

6 F J CORBATO V A VYSSOTSKY

Introduction and overview of the MULTICS system

Proceedings AFIPS FJCC Vol 271965 pp 185-196

7 J H SALTZER

Traffic control in a multiplexed computer system

MAC-TR-30 MIT 1966

$8 \mathrm{JW}$ ATWOOD et al
Project SUE status report

CSRG-11 Computer Systems Research Group University of Toronto 1972

$9 \mathrm{~J} \mathrm{~W}$ ATWOOD B L CLARK M S GRUSHCOW R C HOLT J J HORNING K C SEVCIK

Proceedings of session 'Y2

Individual papers Canadian Information Processing Society Conference Montreal 1972

$10 \mathrm{~J} J$ HORNING B RANDELL

Process structuring

CSRG-15 Computer Systems Research Group University of Toronto 1972

11 G H MEALY B I WITT W A CLARK

The functional structure of $O S / 360$

IBM Systems Journal Vol 5 No 11966 pp 2-51

12 A N HABERMANN

Prevention of system deadlocks

Communications of the ACM Vol 12 No 71969 pp 373-385

$13 \mathrm{R}$ C HOLT

On deadlock in computer systems

CSRG-6 Computer Systems Research Group

University of Toronto 1971

14 A SHOSHANI E G COFFMAN

Prevention, detection and recovery from system deadlocks

Technical Report 80 Dept of Electrical Engineering

Princeton University 1969

$15 \mathrm{~K}$ CORBIN et al

A software laboratory preliminary report

Carnegie Mellon University 1971

16 Y VERNER

On process communication and process synchronization

Dept of Computer Science

University of Toronto 1971

17 C A R HOARE

Towards a theory of parallel programming-a preliminary draft

Queen's University Belfast 1971

18 R S FABRY

Preliminary description of a supervisor for a machine oriented around capabilities

ICR Quarterly Report No 18 Institute for Computer Research University of Chicago 1968

19 G S GRAHAM

Protection structures in operating systems

Dept of Computer Science

University of Toronto 1971

$20 \mathrm{~W}$ A WULF et al

BLISS reference manual

Computer Science Dept

Carnegie Mellon University 1970

21 N WIRTH

PL360-A programming language for the 360 computers Journal of the ACM Vol 15 No 11968 pp 37-74

$22 \mathrm{~N}$ WIRTH

The programming language PASCAL

Acta Informatica Vol 1 No 11971

23 R D BERGERON J GANNON A VAN DAM

Language for systems development

SIGPI.AN Notices Vol 6 No 91971

24 W M MCKEEMAN J J HORNING

D B WORTMAN

A compiler generator

Prentice Hall 1970 
25 B L CLARK

The design of a system programming language

Dept of Computer Science

University of Toronto 1971

26 B L CLARK J J HORNING

The system language for project $S U E$ SIGPLAN Notices Vol 6 No 91971

27 COMPUTATION CENTRE

Efficient production of large programs
Proceedings of International Workshop

Polish Academy of Sciences Jablonna Poland 1970 p 81

28 F T BAKER

Chief programmer team management of production programming

IBM Systems Journal Vol 11 No 11972

29 B H LISKOV

The design of the VENUS operating system

Communications of the ACM Vol 15 No 31972 pp 144-49 